\title{
Ovine neuronal ceroid lipofuscinosis: a large animal model syntenic with the human neuronal ceroid lipofuscinosis variant CLN6
}

\author{
Murray F Broom, Chaoming Zhou, Judith E Broom, Karen J Barwell, Robert D Jolly, \\ Diana F Hill
}

\begin{abstract}
The neuronal ceroid lipofuscinoses (NCLs) are a group of inherited degenerative neurological diseases affecting children. A number of non-allelic variants have been identified within the human population and the genes for some of these have recently been identified. The underlying mechanism for the neuropathology remains an enigma; however, pioneering studies with the naturally occurring ovine model (OCL) have led to the proposal that these diseases represent lesions in specific hydrophobic protein degradation pathways. In this study, we show linkage between OCL and microsatellite markers on OAR 7q13-15. Using interspecies chromosome painting we establish that OAR 7q13-15 is syntenic with human chromosome 15q21-23, the region which was recently defined as the location of a newly identified late infantile variant (CLN6). We propose that our ovine model represents a mutation in the gene orthologous to that mutated in the human late infantile variant CLN6. The ovine linkage flock, consisting of 56 families, represents a powerful resource for positional cloning of this NCL gene. The availability of stich a large animal model will have important implications for experimentation in downstream corrective therapies.

$(\mathcal{O}$ Med Genet 1998;35:717-721)
\end{abstract}

Keywords: neuronal ceroid lipofuscinosis; Batten disease; ovine; animal models

Molecular Biology Unit, Department of Biochemistry,

University of Otago, Box 56, Dunedin, New Zealand

M F Broom

C Zhou

J E Broom

K J Barwell

D F Hill

Department of Veterinary Pathology, Massey University, Palmerston North, New Zealand

R D Jolly

Correspondence to: Dr Broom.

Received 4 December 1997 Revised version accepted for publication 17 February 1998 infantile NCL (CLN2) is localised to $11 \mathrm{p}^{2}$ and a recent report indicates a deficiency of a pepstatin insensitive lysosomal peptidase. ${ }^{3} \mathrm{Ju}-$ venile NCL (CLN3) is localised to chromo- some $16 \mathrm{p} 12$ and the associated gene codes for a novel putative membrane protein. ${ }^{4}$

A number of human variants have been identified by linkage analysis. The specific disease causing mutations in these syndromes remain unresolved. These include two late infantile forms, CLN5 and CLN6, localised to chromosomes $13 q 22^{5}$ and $15 q 21-23,{ }^{2}$ respectively. A variant disease with juvenile onset is linked to chromosome $1 \mathrm{p} 32$ and has recently been shown to be biochemically analogous to INCL. ${ }^{6}$ In addition to the above entities, adult onset forms of ceroid lipofuscinosis are also known.

Despite recent advances in molecular genetics, the pathogenesis of the ceroid lipofuscinoses remains enigmatic. The function of PPT in INCL is uncertain as is the role of the CLN3 protein in JNCL. Attempts to develop mouse knockout models of CLN1 and CLN3 have so far proven to be unsuccessful. Furthermore, it is becoming increasing clear that in many instances rodent models of human disease show significantly altered pathologies. ${ }^{7-9}$

Well defined animal models that mimic the human disease phenotype are important resources for biochemical and therapeutic studies. The best described animal model of NCL occurs in South Hampshire sheep (OCL) and has been extensively studied as a model of the human diseases. ${ }^{10}$ Tissue extracts from the OCL sheep ${ }^{11-13}$ contain multilamellar storage material and provided the first evidence that subunit $c$ of the mitochondrial ATP synthase accumulates in storage bodies. Subsequent studies showed that the subunit $\mathrm{c}$ was also stored in many of the human NCL variants. ${ }^{13}$ Subunit $\mathrm{c}$ is an extremely hydrophobic protein which extracts with lipids in chloroform and methanol (2:1) and which in situ forms a transmembrane ion channel as part of the $F_{0}$ complex of mitochondrial ATP synthase. ${ }^{14}$

In this study we show by linkage analysis that OCL is located on sheep chromosome 7q13-15. Using interspecies fluorescent in situ hybridisation and comparative gene mapping data we provide strong evidence for synteny of this locus to HSA $15 \mathrm{q} 21-23$, recently reported as the location of CLN6. The gene for the CLN6 disease variant is as yet unknown. The data strongly suggest that OCL is a model for the human variant late infantile disorder, CLN6.

The sheep model is important for future progress in understanding the disease aetiology and for experimentation in therapeutic intervention. Large numbers of sheep families in which a single founder mutation is segregating 
Table 1 Two point lod scores between OCL and chromosome 7 markers

\begin{tabular}{lrrrrrrrrrr}
\hline \multicolumn{1}{c}{ Recombination fraction $(r)$} & & & & & \\
\cline { 2 - 9 } Marker & \multicolumn{1}{c}{0.001} & 0.01 & 0.05 & 0.1 & 0.15 & 0.2 & 0.25 & 0.3 & $Z$ max & Lod \\
\hline BM3033 & -17.08 & -8.20 & -2.46 & -0.46 & 0.39 & 0.76 & 0.86 & 0.79 & 0.25 & 0.86 \\
BMS2635 & -1.59 & 6.03 & 9.88 & 10.05 & 9.17 & 7.85 & 6.30 & 4.67 & 0.08 & 10.18 \\
BM1237 & 11.11 & 10.90 & 9.92 & 8.67 & 7.38 & 6.07 & 4.76 & 3.49 & 0.0 & 11.14 \\
BMS528 & 3.62 & 6.40 & 7.51 & 7.14 & 6.36 & 5.39 & 4.32 & 3.23 & 0.05 & 7.51 \\
BMS2349 & -3.28 & 0.58 & 2.77 & 3.20 & 3.10 & 2.77 & 2.31 & 1.79 & 0.11 & 3.20 \\
BMS861 & -16.61 & -8.70 & -3.49 & -1.58 & -0.68 & -0.20 & -0.05 & 0.16 & 0.34 & 0.18 \\
\hline
\end{tabular}
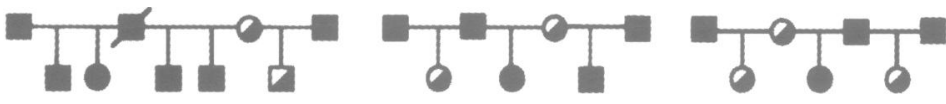

Figure 1 Pedigrees of OCL families and the segregation of the microsatellite marker BM1237. Carrier ewes were mated to different homozygous rams over successive years. Offspring were diagnosed as affected or normal obligate carriers.

provide an effective resource for linkage mapping and disease gene identification. Strong phenotypic resemblance to the human disease coupled with large animal size and ease of maintenance of the flocks make the OCL sheep an attractive model for NCL research.

\section{Methods}

LINKAGE FLOCK

South Hampshire sheep segregating for OCL were maintained by mating affected homozygous OCL males with heterozygous carrier ewes. Offspring were phenotyped by brain biopsy at birth or by observing clinical symptoms at 9-12 months. Affected rams with a life expectancy of 24 months were mated in their first year whereas carrier ewes were mated to different rams in successive years. The linkage flock consists of 350 DNA samples from 56 half sibships consisting of two to 11 offspring from a given carrier ewe.

BMS2635

BMS528

BM1237

BMS2349

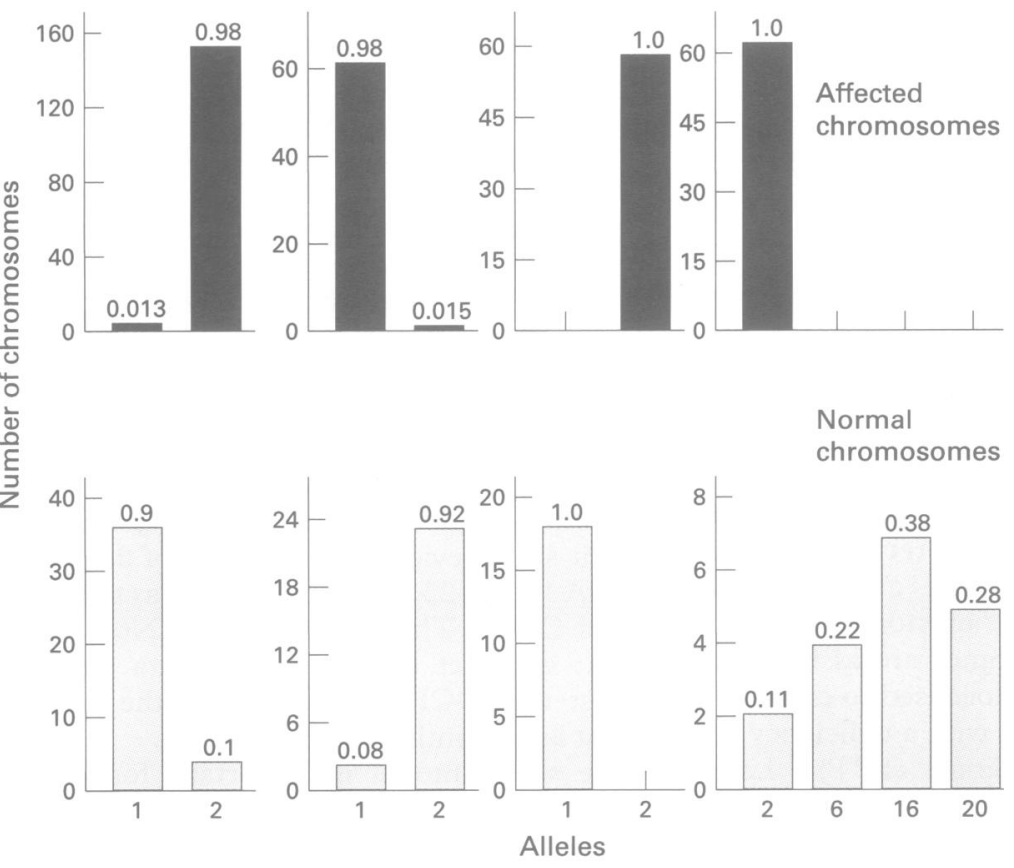

Figure 2 Allelic association of chromosome 7 markers with $O C L$. The allele frequencies for affected and normal chromosomes are shown above each column.
MARKERS

Markers BM3033, BMS2635, BM1237, BMS528, BM2349, TG131, and BM861 are bovine microsatellites. ${ }^{15}$ Markers BM3033, BM1237, BMS528, and BM861 are also framework markers in the sheep linkage map ${ }^{16}$ (Kappes, personal communication).

NOMENCLATURE

BTA, Bos taurus; OAR, Ovis aries; HSA, Homo sapiens; YAC, yeast artificial chromosome; $t_{3}$ Robertsonian $(7 ; 25)$ translocation ${ }^{17}$; DAPI, 4,6 diamidino-2-phenylindole. The main human NCL divisions are infantile (INCL), late infantile (LINCL), and juvenile (JNCL). The loci are designated as follows: CLN1, infantile neuronal ceroid lipofuscinosis; CLN2, classical late infantile neuronal ceroid lipofuscinosis; CLN3, juvenile neuronal ceroid lipofuscinosis; CLN5, variant late infantile neuronal ceroid lipofuscinosis; CLN6 variant late infantile neuronal ceroid lipofuscinosis.

YAC LIBRARY SCREENING

Ovine yeast artificial chromosomes (YACs) were isolated by screening the library pools with microsatellite primers as previously described. ${ }^{18} 19$

\section{IN SITU HYBRIDISATION}

YAC DNA was prepared in agarose plugs and $1 \mu \mathrm{g}$ of extracted DNA was used for standard in situ hybridisation. ${ }^{20}$ Flow sorted sheep chromosome paints have been previously described. ${ }^{21}$ The protocol for hybridisation of sheep chromosome specific paint probes onto human metaphase spreads was modified as follows: the amount of biotin labelled probe was increased to $500 \mathrm{ng}$; after denaturation the probe was preannealed with both sheep $(20 \mu \mathrm{g})$ and human Cot $1(10 \mu \mathrm{g})$ DNA for 4.5 hours $\left(37^{\circ} \mathrm{C}\right.$ in $50 \%$ formamide) in a total of $20 \mu \mathrm{l}$ of hybridisation solution. Before application of the probe to the slide the formamide concentration was lowered to $40 \%$ by the addition of $5 \mu \mathrm{l}$ of $2 \times$ SSC. The probe was hybridised for five days at $37^{\circ} \mathrm{C}$. The stringency washes were performed using $3 \times$ five minute washes in $44 \%$ formamide $/ 2 \times \mathrm{SSC}$ at $37^{\circ} \mathrm{C}$ and $3 \times$ five minutes in $0.5 \times \mathrm{SSC}$ at $50^{\circ} \mathrm{C}$. Development of the signal with FITC streptavidin and biotinylated anti-avidin followed standard protocols. The images were captured using a PXL1400 cooled CCD Camera (Photometrics) attached to a Zeiss Axiophot fluorescent microscope. The images were processed using Quips Smart Capture FISH and CGH software from Vysis in conjunction with IPLAB scientific imaging software from Signal Analytics running on a Power Macintosh 8100/100.

LINKAGE MAPPING

Ovine and bovine microsatellite markers were selected from the developing sheep and cattle maps. ${ }^{15}{ }^{16}$ Where possible, markers selected at approximately 10-15 cM were tested for linkage to OCL. Markers were initially screened using approximately 90 DNA samples; areas of potential interest were then further investigated with more families and, 


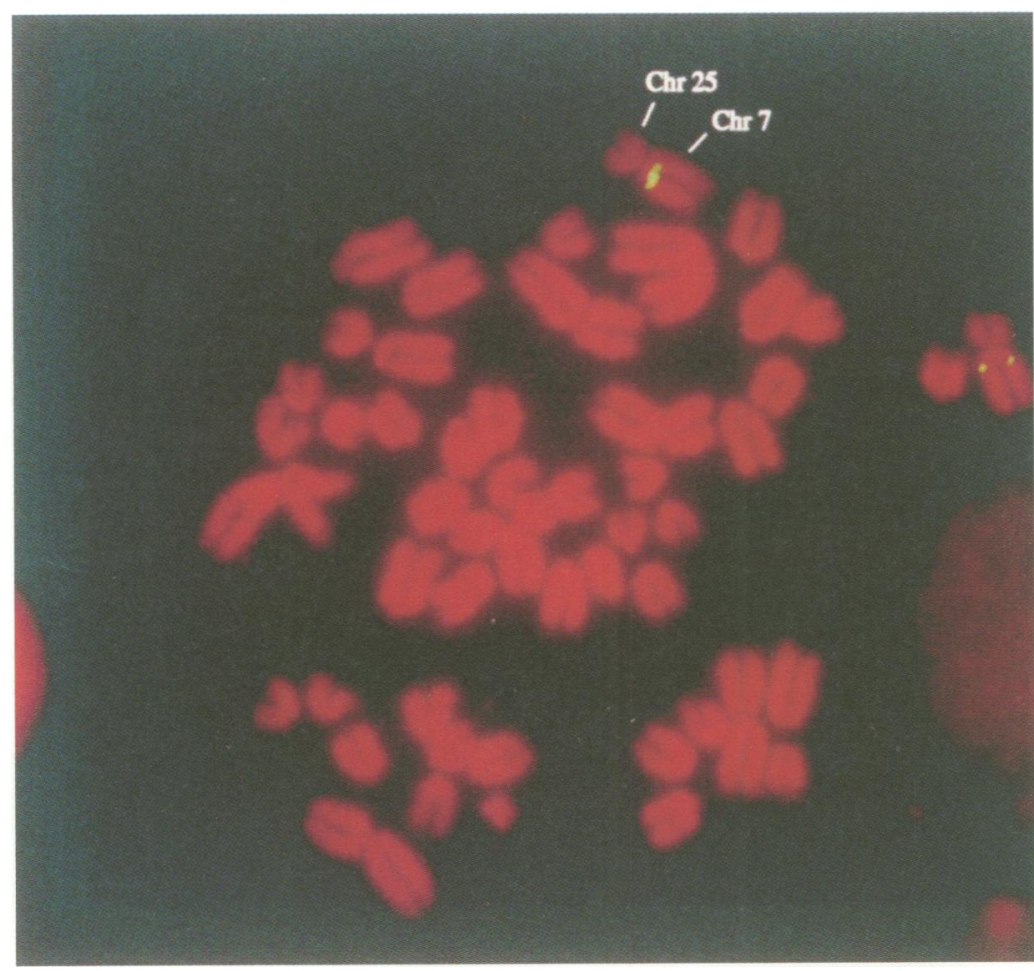

Figure 3 In situ hybridisation of YAC clone 354D3 to an ovine metaphase spread from a homozygous $t_{3}$ Robertsonian translocation: a fusion of ovine chromosome 7 and chromosome 25 .

where possible, additional markers. A total of 160 microsatellite markers were analysed. After identification of a linked marker, flanking markers were identified from the ovine and bovine maps and tested for linkage to OCL. Two point lod scores were determined using CRI-MAP ${ }^{22}$ and multipoint linkage analysis was performed using CRI-MAP Build.

\section{Results}

The frequency of affected offspring in the OCL flock was approximately 0.5 and the uniformity of the phenotype is consistent with a single founder mutation. The microsatellite marker
BMS528 was the first marker analysed to show significant linkage to OCL. Screening of adjacent microsatellite markers (BMS2635, BM1237, BMS2349) from the bovine linkage map $^{15}$ confirmed the location of OCL on sheep chromosome 7 . The segregation of microsatellite marker BM1237 in three families is shown in fig 1. A maximum pairwise lod score of 11.14 was obtained at $\mathrm{Zmax}=0$ between BM1237 and OCL (table 1). Significant linkage was also detected with microsatellite markers BMS2635, BMS528, and BMS2349. Multipoint linkage analysis placed OCL in the region of BMS1237 (fig 2). Strong allelic association was observed with markers BMS2635, BM1237, BMS528, and BMS2349. The allelic association was the strongest at BM1237; all affected chromosomes carry allele 2 and all normal chromosomes carry allele 1 (fig 2 ).

YAC clones containing microsatellite markers BMS2635, BM1237, BMS528, TGLA131, and BMS2349 were used for in situ hybridisation. Identification of sheep chromosome 7 as the target chromosome was simplified by the use of a homozygous Robertsonian $t_{3}$ translocation consisting of a 7/25 centromeric fusion. ${ }^{17}$ Non-chimeric YAC clones consistently hybridised to the chromosome 7 in the region of q13-15. Fig 3 shows the hybridisation of YAC clone 354D 3 to the chromosome $7 \mathrm{arm}$ of the $7 ; 25$ translocation karyotype.

Direct demonstration of synteny between sheep chromosome 7 and human chromosome $15 q$ was obtained by interspecies FISH. Sheep chromosome 7 specific paint probes ${ }^{21}$ were hybridised onto human metaphase spreads and both HSA 15 and 14 were consistently targeted. DAPI banding enhanced by Smart Capture software showed that the signal from the sheep chromosome 7 paint covered the region HSA 15q21-23 (fig 4).
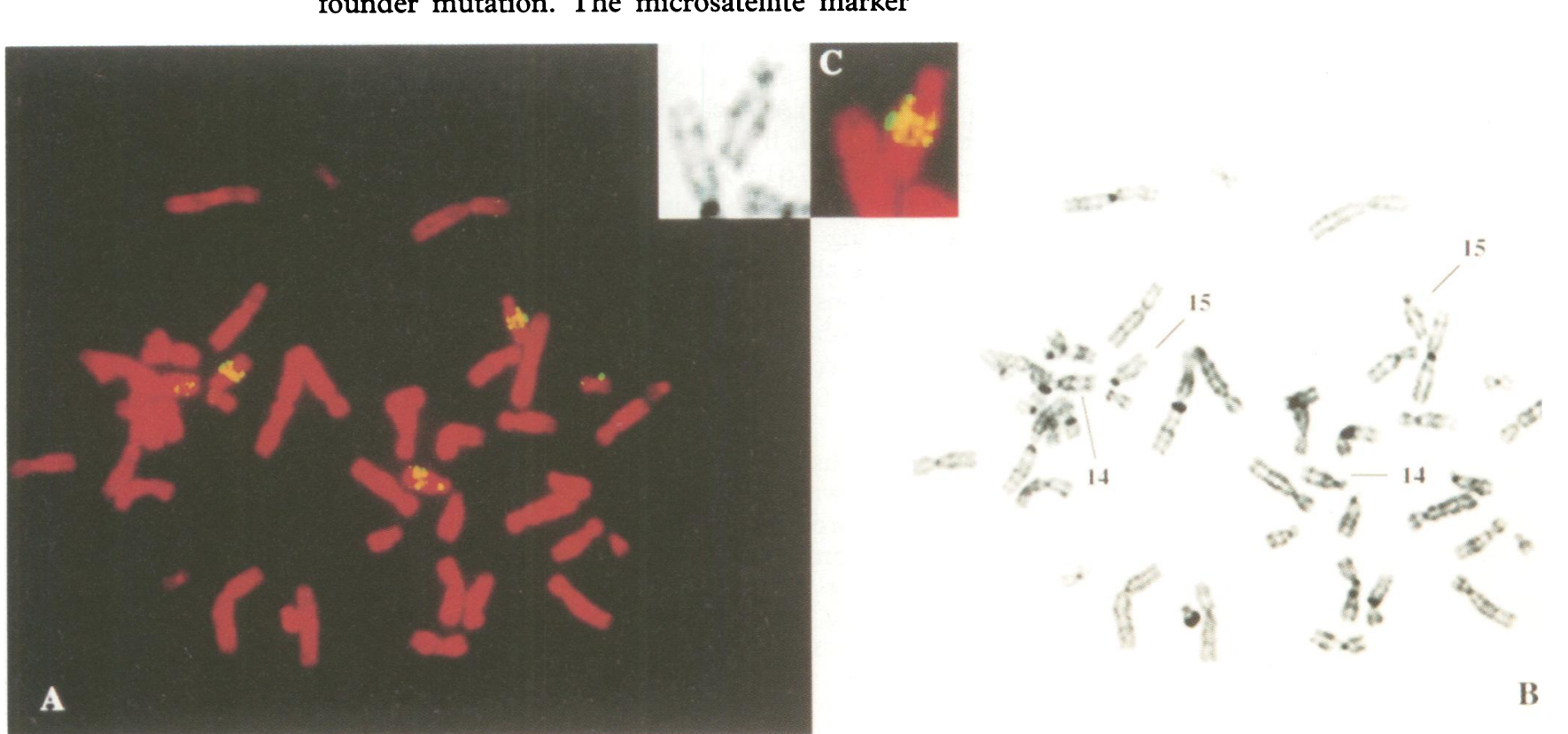

Figure 4 In situ hybridisation of ovine chromosome 7 paint probe onto a human metaphase spread. (A) Hybridisation of paint probe onto DAPI stained human metaphase chromosomes. (B) DAPI banding pattern of the metaphase chromosomes with arrows indicating human chromosomes 14 and 15 . (C) Magnified image of human chromosome 15 showing the DAPI banded image and the fluorescence signal. 


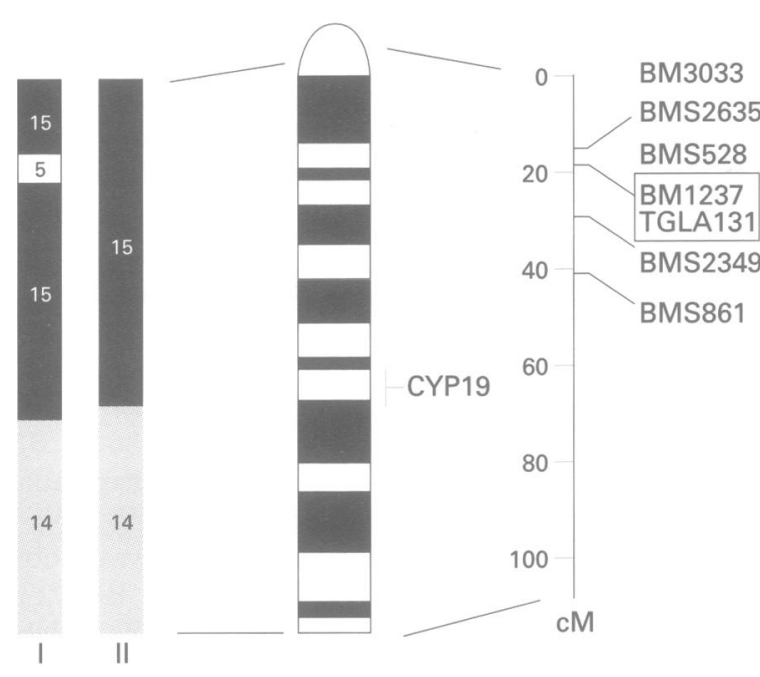

BTA 10

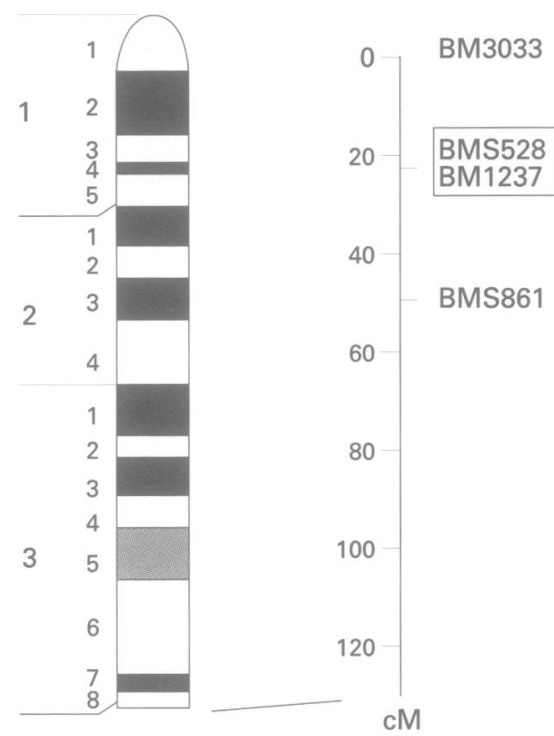

OAR 7

A

B

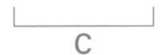

D

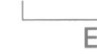

Figure 5 Syntenic relationships between HSA15, BTA10, and OAR7. Section A (I from reference 24, II from reference 23) summarises two previous chromosome painting studies showing the hybridisation of human paint probes (HSA chromosomes 14, 15, and 5) onto bovine chromosome 7. Sections B and D: ideograms of G banded BTA10 and OAR7. Section C: bovine linkage map showing markers ${ }^{15}$ that were informative in the OCL critical region (distances sex averaged). The genetic distances between BM1237 and BMS528 is $4.3 \mathrm{cM}$ (sex averaged). Section E: sheep linkage map showing marker distances (sex averaged) from the International Mapping flock ${ }^{16}$ (Kappes, personal communication). Markers BM1237 and BMS528 were not separable by linkage analysis in the International Mapping flock; however, within the OCL flock the genetic distances between BM1237 and BMS528 was determined to be 4.3 cM (sex averaged).

\section{Discussion}

LINKAGE OF MARKERS TO OCL

A genomic screen of 160 microsatellite markers has led to the identification of four markers linked to OCL. Strong linkage disequilibrium between DCL and markers BMS2635, BM1237, BMS528, and BMS2349 was observed with the strongest allelic association shown between OCL and BM1237.

USING COMPARATIVE MAPPING TO ESTABLISH SYNTENY OF CLN6 WITH OCL

CLN6 has recently been assigned by linkage analysis to HSA $15 \mathrm{q} 21-23 .^{2}$ Several lines of evidence indicate that the OCL and CLN6 regions are syntenic. Firstly, YAC clones containing markers linked to OCL hybridised to the sheep chromosome region $7 q 13-14$. We used a sheep chromosome 7 specific paint probe for interspecies painting onto human metaphase spreads. The sheep chromosome 7 paint hybridised onto regions of human chromosomes 14 and 15. Enhanced DAPI banding showed that the fluorescent signal on HSA chromosome 15 extended from 15q21 to $15 q 23$ (fig 5C).

Secondly, two previously published chromosome painting studies have shown that human chromosomes 14 and 15 paint probes hybridise to cattle chromosome 10 (summarised in fig 5A, B). The HSA 15 paint probe hybridised to BTA $10 \mathrm{q} 11-26^{23} 24$ and synteny between this region in cattle and sheep chromosome 7q11-24 is firmly established. ${ }^{15} 172526$

Thirdly, hexosaminodase A (alpha polypeptide, GDB:120040) maps to HSA 15q23-24 and OAR 7q12-13 and cytochrome P450 aromatase (GDB:119830), which maps to HSA
$15 \mathrm{q} 21$, has been assigned to cattle chromosome $10^{27}$ and sheep chromosome $7 .^{28}$

CLASSIFICATIONS OF NCL

The South Hampshire sheep has been pivotal to our present understanding of this group of diseases. ${ }^{132}$ As a result we can now divide these diseases into at least two groups, those that accumulate subunit $c$ of the mitochondrial ATP synthase as a dominant chemical species in storage material and those that do not. The former group show a variety of multilamellar profiles in storage material on electron microscopy which are of some nosological significance. This group comprises classical (CLN2) and variant LINCLs (CLN5, CLN6), ${ }^{30}{ }^{31}$ JNCL, ${ }^{30}$ and probably adult NCL in humans. ${ }^{32}$

The second group of diseases in which storage of subunit $\mathrm{c}$ is not observed comprises INCL and a juvenile onset variant NCL. These diseases typically show granular osmiophilic deposits (GRODS) in lysosomes when examined by electron microscopy. ${ }^{33}$

OVINE NCL: ITS CONTRIBUTION, PAST, PRESENT, AND FUTURE

Although the detailed biochemistry and neurobiology of the ceroid lipofuscinoses remains unresolved, rapid progress is being made using positional cloning. The demonstration of synteny between the OCL and CLN6 chromosomal regions supports the hypothesis that OCL and CLN6 are caused by mutations in orthologous genes. There are at least four nonallelic forms of NCL in humans that show lysosomal storage of subunit c. It can be expected that these disorders are linked by some common factor; either the gene products 
are part of a specific biochemical pathway or the biochemical lesions disrupt the orderly degradation of subunit $\mathrm{c}$ allowing it to aggregate into complexes with lipids that cannot be catabolised by lysosomal enzymes.

The current study validates further the importance of the sheep model. With its single founder mutation, large flock size, large animal morphology, and fidelity in mimicking the human condition, the sheep model must continue to play an important role in the future for unraveling the aetiology of the NCLs.

We acknowledge the funding of the Health Research Council of New Zealand for MFB and CZ. KJB is a recipient of a University of Otago Postgraduate Scholarship. JEB was a recipient of a University of Otago Fanny Evans Scholarship. We acknowledge financial support from the New Zealand Lottery Grants Board for funding the in situ imaging facility and thermocyclers. RDJ acknowledges financial support from the United States Institute of Neurological Disorder and Stroke, grant numbers NS11238 of Neurological Disorder and Stroke, grant numbers NS11238 typing human chromosomes.

1 Hellsten E, Vesa J, Jalanko A, Peltonen L. From locus to cellular disturbances: positional cloning of the infantile lular disturbances: positional cloning of the infantile
neuronal ceroid-lipofuscinosis gene. Neuropediatrics neuronal ceroid

2 Sharp JD, Wheeler RB, Lake BD, et al. Loci for classical and a variant late infantile neuronal ceroid lipofuscinosis map to chromosomes $11 \mathrm{p} 15$ and 15q21-23. Hum Mol Genet 1997;6:591-5.

3 Sleat DE, Donnelly RJ, Lackland $\mathrm{H}$, et al. Association of mutations in a lysosomal protein with classical lateinfantile neuronal ceroid lipofuscinosis. Science 1997;277. 1802-5.

4 Consortium TIBD. Isolation of a novel gene underlying Batten disease, CLN3. Cell 1995;82:949-57.

5 Klokars T, Savukoski M, Isosomppi J, et al. Efficient construction of a physical map by fibre-fish of the CLN5 region: refined assignment and long range contig covering the critical region on 13q22. Genomics 1996;35:71-8.

6 Mitchison HM, Hofmann SL, Becerra CHR, et al. Mitchison HM, Hofmann SL, Becerra CHR, et al. Mutations in the palmitoyl-protein thioesterase gene (PPT; $C L N 1$ ) causing juvenile ceroid lipofuscinosis with gran
osmiophilic deposits. Hum Mol Genet 1998;7:291-7.

7 Harris A. Towards an ovine model of cystic fibrosis. Hum Mol Genet 1997;6:2191-3.

8 Edwards $\mathrm{JH}$. Comparative genome mapping in mammals. Curr Opin Genet Dev 1994;4:861-7.

9 Hendrickson EA. The SCID mouse: relevance as an animal model system for studying human disease. Am $\mathcal{f}$ Pathol 1993;143:1511-22.

10 Jolly RD, Martinus RD, Palmer DN. Sheep and other animals with ceroid-lipofuscinoses: their relevance to Batten disease. Am ₹ Med Genet 1992;42:609-14.

11 Palmer D, Martinus R, Cooper S, Midwinter G, Reid J, Jolly R. Ovine ceroid lipofuscinosis: the major lipopigment protein and the lipid-binding subunit of mitochondrial ATP synthase have the same $\mathrm{NH}_{2}$-terminal sequence. $\mathcal{F} \mathrm{Biol}$ synthase have the same
Chem $1989 ; 264: 5736-40$.

12 Fearnley IM, Walker JE, Martinus RD, et al. The sequence of the major protein stored in the ovine ceroid lipofuscinosis is identical with that of the dicyclohexylcarbodiimidereactive proteolipid of mitochondrial ATP synthase. Biochem $\mathcal{F}$ 1990;268:751-8.

13 Jolly RD. The ovine model of ceroid-lipofuscinosis (NCL): its contribution to understanding the pathogenesis of Batten disease. Neuropediatrics 1997;28:60-2.
14 Nijtmans LGJ, Klement P, Houstek J, van den Bogert C. Assembly of mitochondrial ATP synthase in cultured human cells: implications for mitochondrial diseases. Biochim Biophys Acta 1995;1272:190-8.

15 Kappes SM, Keel JW, Stone RT, et al. A second-generation linkage map of the bovine genome. Genome Res 1977;7 235-49.

16 Crawford AM, Dodds KG, Ede AJ, et al. An autosomal genetic linkage map of the sheep genome. Genetic 1995;140:703-24.

17 Ansari HA, Pearce PD, Maher DW, Malcolm AA, Broad TE. Resolving ambiguities in the karyotype of domestic sheep (Ovis aries). Chromosoma 1993;102:340-7.

18 Broom MF, Hill DF. Construction of a large-insert yeast artificial chromosome library from sheep DNA. Mammalian Genome 1994;5:817-19.

19 Bentley DR, Todd C, Collins J, et al. The development and application of automated gridding for efficient screening of yeast and bacterial ordered libraries. Genomics 1992;12: 534-41.

20 Tebbutt SJ, Broom MF, van Stijn TC, Montgomery GW, Hill DF. Genetic and physical mapping of the ovine cystic fibrosis gene. Cytogenet Cell Genet 1996;74:245-7.

21 Burkin DJ, O'Brian PCM, Broad TE, et al. Isolation of chromosome-specific paints from high-resolution flow karyotype of the sheep (Ovis aries). Chrom Res 1997;5:1-7.

22 Lander ES, Green P. Construction of multilocus genetic linkage maps in humans. Proc Natl Acad Sci USA 1987;84: 2363-7.

23 Chowdhary BP, Fronicke L, Gustavsson I, Scherthan H. Comparative analysis of the cattle and human genomes: detection of ZOO-FISH and gene mapping-based chromosomal homologies. Mammalian Genome 1996;7:297-302.

24 Solinas-Toldo S, Lengauer C, Fries R. Comparative genome map of human and cattle. Genomics 1995;27:489-96.

25 DiBerardino D, Hayes H, Fries R, Long S. Internationa system for cytogenetic nomenclature of domestic animals (1989). Cytogenet Cell Genet 1990;53:65-79.

26 Popescu CP, Long S, Riggs P, et al. Standardization of cattle karyotype nomenclature: report of the committee for the standardization of the cattle karyotype. Cytogenet Cell Genet 1996;74:259-61.

27 Goldammer T, Guerin G, Brunner RM, Vanselow J, Furbass R, Schwerin M. Chromosomal mapping of the bovine aromatase gene (cyp19) and an aromatase pseudogene to chromosome 10 and syntenic group U5. Mammalian Genome 1994;5:826-7.

28 Payen E, Saidi-Mehtar N, Pailhoux E, Cotinot C. Sheep gene mapping: assignment of ALDOB, CYP19, WT and SOX 2 by somatic cell hybrid analysis. Animal Genet 1995; 26:331-3.

29 Palmer DN, Jolly RD, van Mil HC, Tyynela J, Westlake VJ. Different patterns of hydrophobic protein storage in different forms of neuronal ceroid-lipofuscinosis (NCL, Batten disease). Neuropediatrics 1997;28:45-8

30 Palmer DN, Fearnley IM, Medd SM, et al. Lysosomal storage of the DCCD reactive proteolipid subunit of mitochondrial ATP synthase in human and ovine ceroid lipofuscinoses. In: Porta EA, ed. Lipofuscin and ceroid pigments. Vol 1. New York: Plenum Press, 1990:211-23.

31 Tyynela J, Suopanki J, Santavuori P, Baumann M, Haitia M. Variant late infantile ceroid-lipofuscinosis: patholmorphology and biochemistry. F Neuropathol Exp Neurol 1997;56: 369-75.

32 Westlake VJ, Jolly RD, Bayliss SL, Palmer DN. Immunocytochemical studies in the ceroid-lipofuscinoses (Batten disease) using antibodies to subunit $c$ of the mitochondrial ease) using antibodies to subunit $c$ of the mito

33 O'Rawe AM, Mitchison HM, Williams R, et al. Molecular genetic analysis of a variant form of Batten disease with granular osmiophilic deposits. Sixth International Congres on Neuronal Ceroid-Lipofuscinoses, Gustavelund, Finland, 1996:Abstract 17 